\title{
Topological surface state in epitaxial zigzag graphene nanoribbons
}

\author{
Thi Thuy Nhung Nguyen, ${ }^{\dagger}$ Niels de Vries, ${ }^{\ddagger}$ Hrag Karakachian, "Markus \\ Gruschwitz, $^{\dagger}$ Johannes Aprojanz, $^{\dagger}$ Alexei A. Zakharov, ${ }^{\S}$ Craig Polley ${ }^{\S}$ Thiagarajan \\ Balasubramanian, ${ }^{\S}$ Ulrich Starke, ${ }^{\Uparrow}$ Cornelis F.J. Flipse, ${ }^{\ddagger}$ and Christoph \\ Tegenkamp*,† \\ $\dagger$ Institute for Physics, Technical University of Chemnitz, 09126 Chemnitz, Germany \\ $\ddagger$ Faculty of Applied Physics, Eindhoven University of Technology, Groene Loper 19, 5612 \\ AP Eindhoven, The Netherlands \\ 【Max Planck Institute for Solid State Research, Heisenbergstraße 1, 70569 Stuttgart, \\ Germany \\ $\S M A X I V$ Laboratory and Lund University, 22100 Lund, Sweden \\ E-mail: christoph.tegenkamp@physik.tu-chemnitz.de \\ Phone: +49 (0)371 531-33103. Fax: +49 (0)371 531-833103
}

The supplementary information contains details about:

1. High resolution low energy electron diffraction (SPALEED) measurements

2. Angle resolved photoelectron spectroscopy (ARPES)

3. Raman measurements

4. Tight binding calculations

5. Bulk band gap

6. Transmission electron microscopy (TEM) measurements 


\section{High resolution low energy electron diffraction (SPALEED)}

\section{measurements}

The successful combination of lithography for the mesas and subsequent selective growth on the facets is nicely demonstrated by electron diffraction. Representative high resolution LEED patterns using SPALEED (spot profile analysis low energy electron diffraction) are depicted in Fig. S1a) and b) taken at a primary energy of $190 \mathrm{eV}$ and $175 \mathrm{eV}$, respectively. Both patterns exhibit first order diffraction spots of $\mathrm{SiC}$ (dark blue) and graphene (red) in coexistence with the $(6 \times 6)$ (light blue) and $(6 \sqrt{3} \times 6 \sqrt{3}) \mathrm{R} 30^{\circ}$ (purple) reconstruction spots. The latter are characteristic for the buffer layer on SiC. ${ }^{1}$ In addition to the wellknown spots from the (0001) plane, there are spots that change their positions as a function of energy in reciprocal space indicating diffraction from inclined surfaces, i.e. from facets on the sidewalls. ${ }^{[2}$ The facet spots appear on both sides of each $\mathrm{SiC}$ basal spot and move along the $[11 \overline{2} 0]_{\mathrm{SiC}}$ direction which is indicative for sidewalls aligned parallel to the $[1 \overline{1} 00]_{\mathrm{SiC}}$ direction with opposite inclinations. It should be noted that the appearance of the facet spots in close vicinity to the first order diffraction spots of $\mathrm{SiC}$ suggests that the electron diffraction experiments probe the underlying $\mathrm{SiC}$ facet structure rather than the $\mathrm{zz}$-GNRs on top of the sidewalls. The transfer width of the SPA-LEED system is close to the pitch size of mesa structure, i.e. the signals from the facets are incoherently collected from around $10^{4}$ ribbons.

In order to get a deeper insight into the nature of the SiC sidewalls and quantify the long range order of the $\mathrm{SiC}$ facets hosting the ribbons, so-called $\left(\mathrm{k}_{\perp}, \mathrm{k}_{\|}\right)$reciprocal space maps (RSMs) were taken. These maps consist of spot profiles taken along the $[11 \overline{2} 0]_{\mathrm{SiC}}$ direction (cf. orange spot profiles in Fig. $\left[\mathrm{S1}_{\mathrm{a}}\right)$ and b)) at various electron energies $E$. The $\left(\mathrm{k}_{\perp}, \mathrm{k}_{\|}\right)$RSM shown in Fig. S1 ) clearly displays two sets of Ewald rods. The rods parallel to the scattering phase axes, which is related to the energy by $S=k_{\perp} d / 2 \pi=2 d \sqrt{E(\mathrm{eV}) / 150.4}$, originate from the (0001) plane, while the inclined rods reflect the energy dependent positions 
of the facet spots in reciprocal space. ${ }^{213}$ The facet angle can be accurately determined from the RSM and amounts here to $\alpha=27^{\circ}$. Interestingly, the facet rods intersect the basal rods three times within one full-phase shift of $\Delta S=1$. Considering that the scattering phase $S$ was defined with respect to a monoatomic $6 \mathrm{H}-\mathrm{SiC}$ step with a height of $\mathrm{d}=0.25 \mathrm{~nm}$, the presence of crossings at out-of-phase conditions suggests the existence of multi-atomic steps on the sidewalls. $\frac{214}{}$ According to $S=k_{\perp} d / 2 \pi$, three crossings thus correspond to steps with a height of $0.75 \mathrm{~nm}$, i.e. half a unit cell of $6 \mathrm{H}-\mathrm{SiC}$.
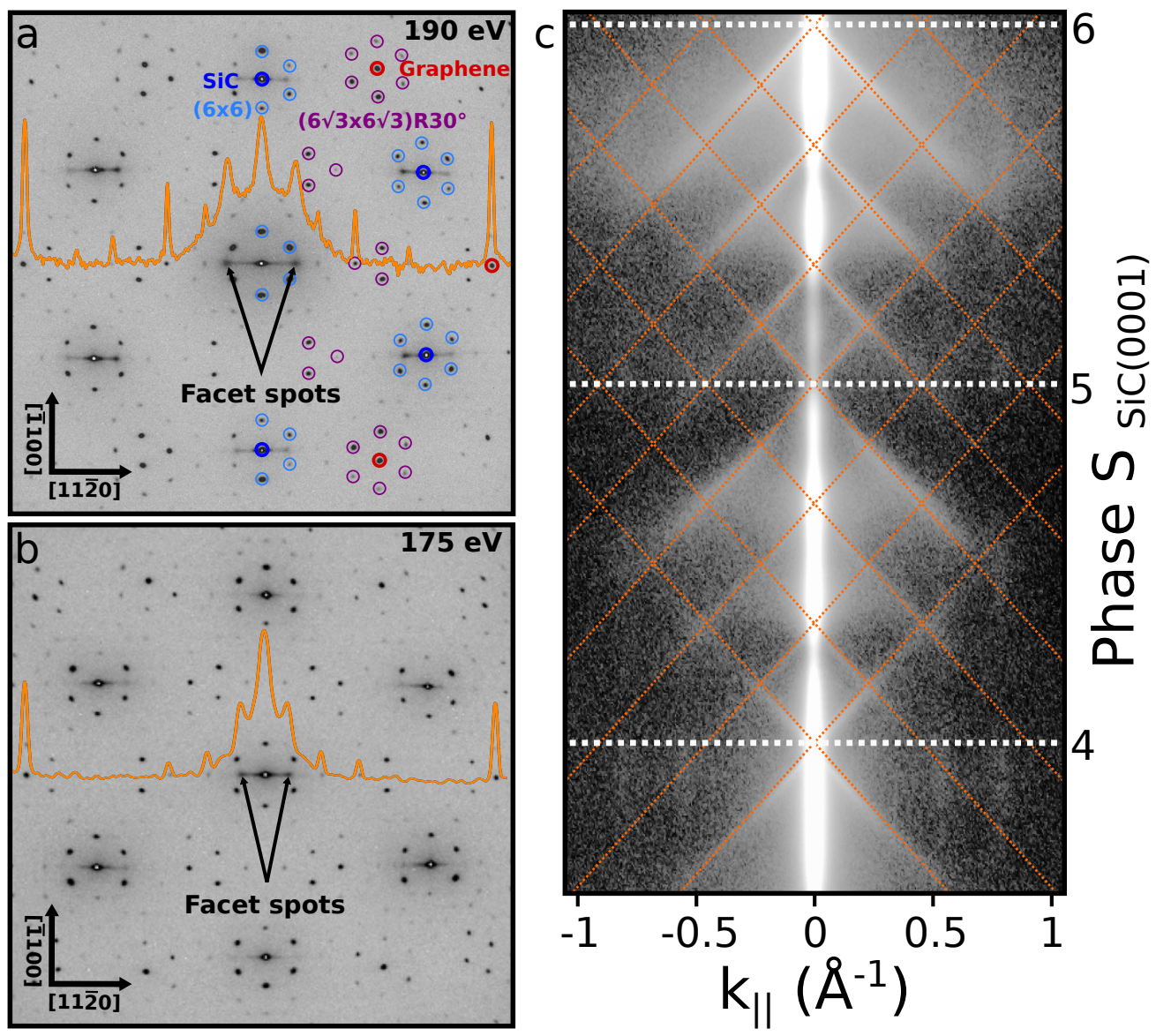

Figure S1: a,b) SPA-LEED patterns taken at $190 \mathrm{eV}$ and $175 \mathrm{eV}$. The patterns exhibit the coexistence of first order diffraction spots of graphene and $\mathrm{SiC}$ as well as buffer layer-related reconstruction spots. The colored symbols are explained in the text. In addition, facet spots occur in the vicinity of the first order spots of $\mathrm{SiC}$, which move with energy in reciprocal space. c) $\left(\mathrm{k}_{\perp}, \mathrm{k}_{\|}\right)$-RSM taken on $\mathrm{SiC}$ with zz-sidewalls along the $[11 \overline{2} 0]_{\mathrm{SiC}}$ direction through the specular spot. The scattering phase $\mathrm{S}$ is defined with respect to a single SiC-bilayer $(d=0.25 \mathrm{~nm})$. 

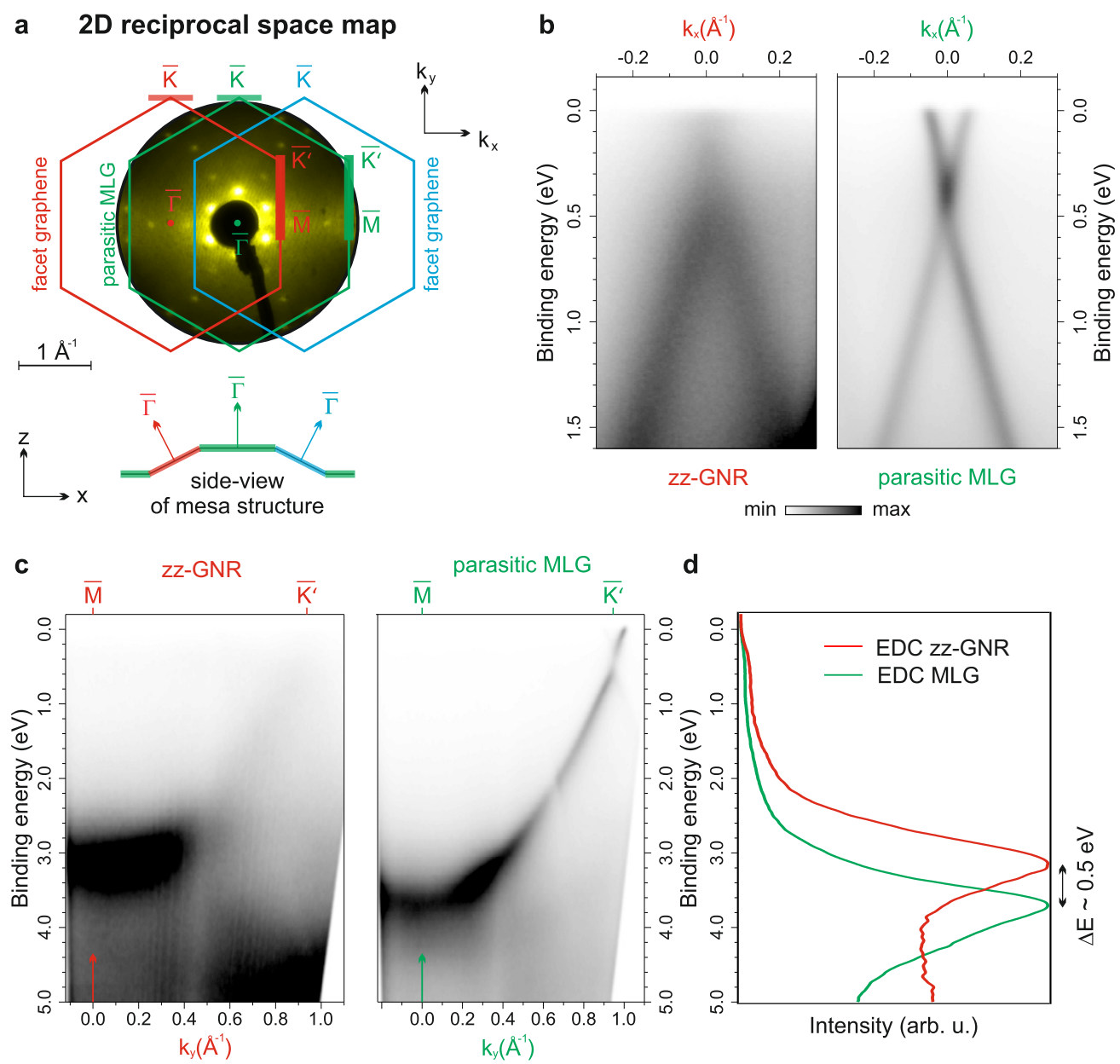

Figure S2: (color online) a) 2D reciprocal space map and side-view of the mesa structure in real space. b) ARPES energy-momentum cuts taken at the $\overline{\mathrm{K}}$-points of zz-GNR and parasitic basal MLG, perpendicular to their respective $\overline{\Gamma K}$-directions. c) ARPES energy momentum cuts along the $\overline{\mathrm{MK}^{\prime}}$-orientations of zz-GNR and parasitic basal MLG. d) Energy distribution curves (EDCs) taken at the $\overline{\mathrm{M}}$-points of both zz-GNR and basal graphene layers, marked by the red and green arrows in c) respectively.

\section{Angle resolved photoelectron spectroscopy, ARPES}

The band structure of the zz-GNRs was investigated by ARPES. Fig. S2a) presents the twodimensional reciprocal space map (2D-RSM) of high symmetry points, which is constructed by superimposing the Brillouin zones (BZs) of the zz-GNRs and of the parasitic MLG on top of the LEED pattern measured at $16.5 \mathrm{eV}$. The amount by which the BZs of GNRs are shifted away from normal emission is given by the angle of inclination relative to the basal plane of the substrate in real space $\left(27^{\circ}\right) .[5$ 
Fig. S2 2 ) represents ARPES energy-momentum cuts taken at the $\overline{\mathrm{K}}$-points of zz-GNR and parasitic MLG, perpendicular to their respective $\overline{\Gamma K}$ directions (the measurement directions are indicated in Fig. $(\mathrm{S} 2 \mathrm{a})$. The $\pi$-band originating from the zz-GNR is clearly resolved. It is far less doped as compared to the parasitic MLG on the basal plane, who's Dirac point is located at $0.43 \mathrm{eV}$ below $\mathrm{E}_{F}$, showing the well-know n-type doping induced by the interfacial carbon buffer layer. ${ }^{116}$ A detailed analysis of the determination of the Dirac points, for both zZ-GNR and parasitic MLG, is presented below. Fig. S2 F) reveals the energy momentum cuts taken along the $\overline{\mathrm{MK}^{\prime}}$-directions of the facet and basal graphene layers. The resepctive EDCs taken at the $\overline{\mathrm{M}}$-points are shown in panel d), which highlight the obvious doping difference of about $0.5 \mathrm{eV}$ between the facet zz-GNRs and the basal parasitic MLG.

Determination of the Dirac points: Fig. S3a) displays the $\pi$-bands with the addition of solid lines tracking the spectral maxima. These were deduced from fitting the momentum distribution curves (MDCs), as exemplarily shown for the zz-GNR graphene in panel b). The linear fits to the MDC peaks of the lower $\pi$-band of parasitic MLG show a crossing point at $\mathrm{E}_{D L}=0.50 \pm 0.01 \mathrm{eV}$ (panel $\mathrm{b}$, red dot). The upper $\pi^{*}$-band on the other hand exhibits a crossing point at $\mathrm{E}_{D U}=0.35 \mathrm{eV}$ (Fig. $[\mathrm{S} 3 \mathrm{~b}$, blue dot). We define the actual Dirac point of parasitic MLG, $\mathrm{E}_{D}=0.43 \mathrm{eV}$ (green dot), as the average value between $\mathrm{E}_{D L}$ and $\mathrm{E}_{D U}$, which is in perfect agreement with previous studies. ${ }^{[7} \mathrm{A}$ similar approach is used to determine the Dirac point of facet zz-GNRs. However, due to lower doping levels and the overall upward shift of the Dirac cone, the $\pi^{*}$-band of zz-GNRs cannot be resolved and appropriately fitted using two separate linear curves. Therefore, only the MDCs of the $\pi$ band are fitted, revealing a lower Dirac point $\left(\mathrm{E}_{D L}\right)$ located at $0.11 \mathrm{eV}$ below $\mathrm{E}_{F}$. Comparing the $\mathrm{E}_{D L}$ values extracted from the different $\pi$-bands, we find a doping difference of about $0.4 \mathrm{eV}$ between zz-GNRs and parasitic MLG.

Quantum well states and band gap: In contrast to arm-chair GNR structures on $\mathrm{SiC}$, the ARPES spectra from the zz-GNR reveal no clear signs of quantum well states (QWS). ${ }^{8}$ As the zz-GNR structures on $\mathrm{SiC}$ are wider by one order of magnitude compared to the ac- 
GNR structures, the splitting of the QWS is much smaller. In addition, taking into account a variation of the GNR width along the ribbon direction, the features are smeared out. Further details are discussed below in context of Fig. S10. Due to the growth mode of zz-GNRs, only the lower edge merging perpendicularly into the $\mathrm{SiC}$ substrate is atomically smooth, while the upper edge of the GNR easily meanders. Due to small variations of the facet inclinations and the comparably low edge densities of the GNRs of around $10^{-3}$ monolayer, further details close to the Fermi-level are difficult to resolve. For instance, energy dispersionless electron states along the GNR longitudinal direction, e.g. as reported by Miettinen et al., ${ }^{9}$ were not found in our analysis and finally the exact determination of a potential band gap from these ARPES data is not possible.

In summary, the detailed analysis of the photoemission spectra taken at the $\overline{\mathrm{K}}$ - and $\overline{\mathrm{M}}$ points reveal doping differences of about 0.4 and $0.5 \mathrm{eV}$ respectively as compared to the basal parasitic MLG.Thus, an average shift of $(0.45 \pm 0.05) \mathrm{eV}$ can be estimated, which is in agreement with the STS data presented in the main text, and supports the conclusion that charge neutral zz-GNRs are grown on the sidewalls of SiC mesa structures. 

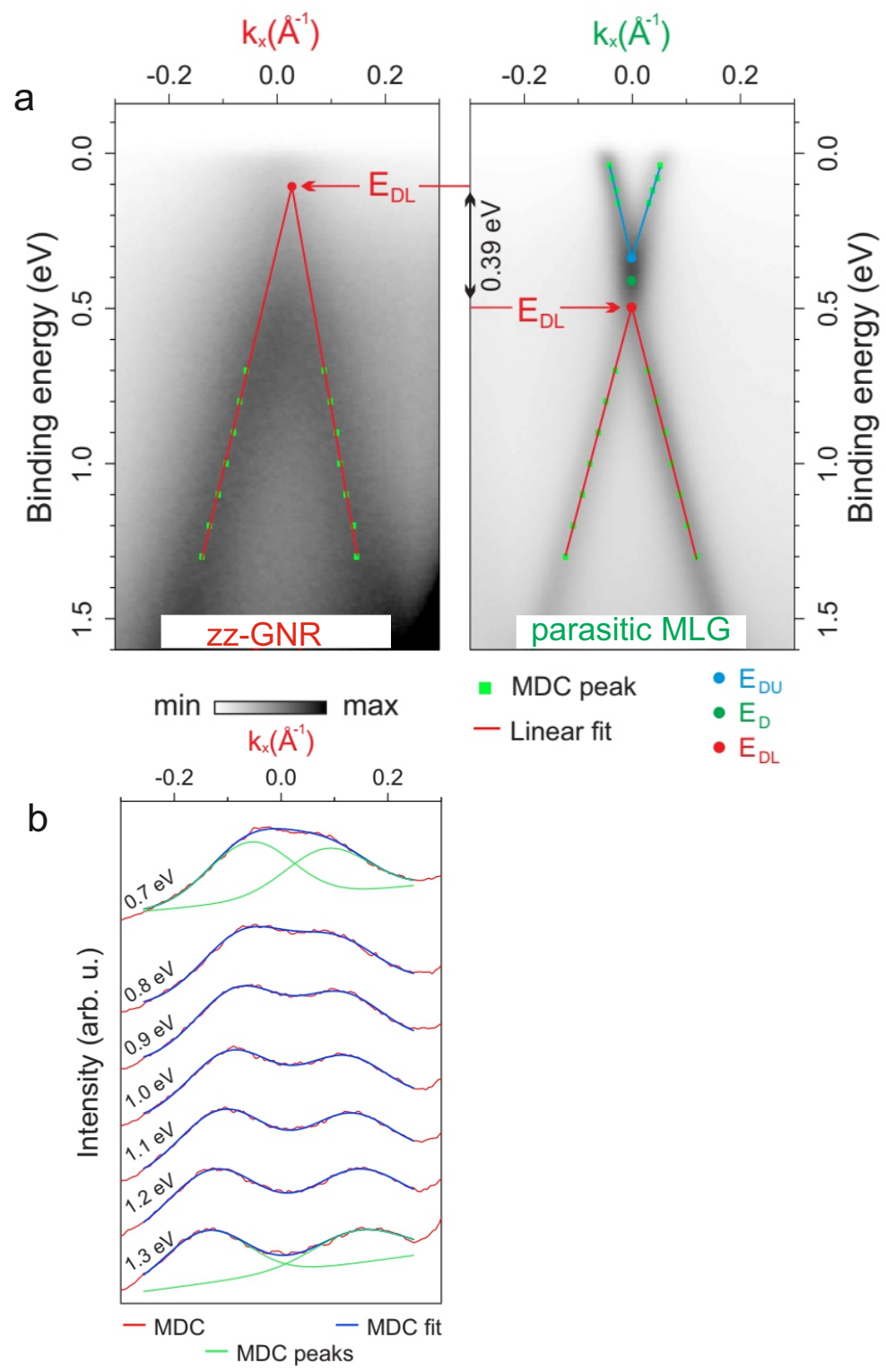

Figure S3: (color online) a) ARPES energy-momentum cuts of the $\pi$-bands taken at the $\overline{\mathrm{K}}$ points of zz-GNR and parasitic basal MLG, perpendicular to their respective $\overline{\Gamma K}$-directions. The linear fits to the band courses and the respective Dirac points extracted from these fits are superimposed on the measured spectra. b) Gaussian fits to the MDCs, exemplarily shown for the zz-GNR. The squared (green) boxes in panel b) refer to the peak maxima. Contrary to the parasitic graphene, this works up to $0.7 \mathrm{eV}$, then the band positions merge. 


\section{Raman measurements}
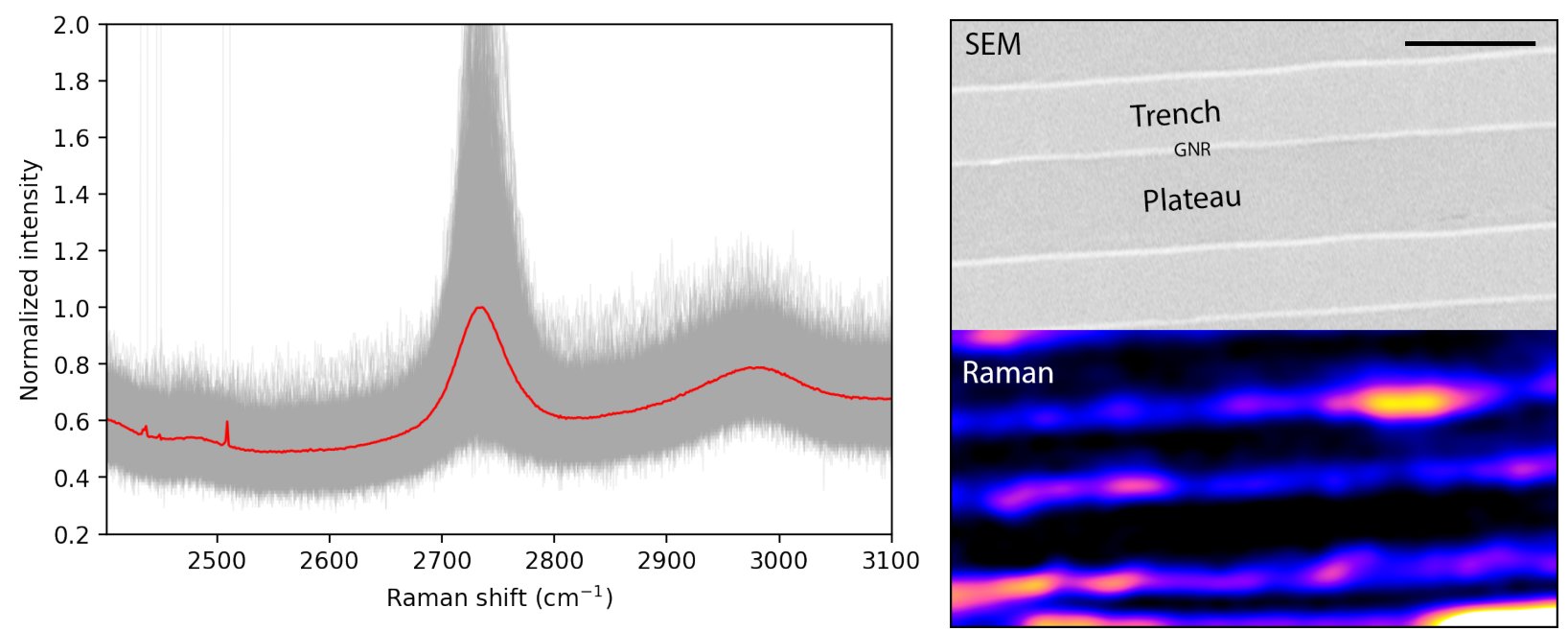

Figure S4: (color online) Raman measurements recorded from a sidewall zz-GNR sample on a $37 \times 37$ grid spanning $80 \times 80 \mu \mathrm{m}$ with an excitation wavelength of $514.5 \mathrm{~nm}$. a) The Raman spectra between 2400 and $3100 \mathrm{~cm}^{-1}$ in grey, their mean in red. In most of the spectra, a peak at $2750 \mathrm{~cm}^{-} 1$ is observed, which is attributed to the graphene $2 \mathrm{D}$ peak. b) The intensity integrated over the range between 2700 and $2800 \mathrm{~cm}^{-1}$ is plotted on the grid, shown in the lower half of the image. A line structure is observed, which corresponds to the geometry of the sample, which is shown with the SEM measurement shown in the top half. The high intensity points in SEM correspond to the sidewall GNRs, which exhibit a Raman signal originating from the 2D peak, whereas the rest of the surface mainly consists of buffer layer graphene, which does not show any Raman intensity between 2700 and $2800 \mathrm{~cm}^{-1}$ shift. The data have been interpolated with a Lanczos algorithm, the scale bar corresponds to $5 \mu \mathrm{m}$.

The Raman spectra were obtained with a Renishaw inVia confocal Raman microscope on a similarly prepared sample with a larger pitch were performed. The measurements were performed using a $514.5 \mathrm{~nm}$ laser, focused through a 100x Leica objective, diffracted with a $1800 \mathrm{~mm}^{-1}$ grating. The laser power was $1 \mathrm{~mW}$ for all the measurements, whereas the spot size is $0.9 \mu \mathrm{m}$. The analysis of these measurements is focused on the $2 \mathrm{D}$ peak of graphene, which is a peak observed in the Raman spectrum of graphene at a shift of $2680 \mathrm{~cm}^{-1}$. Fig. S4a) shows Raman spectra obtained on a $37 \times 37$ point grid spanning a $80 \times 80 \mu \mathrm{m}$ area are shown. Most of the spectra exhibit a peak at $2750 \mathrm{~cm}^{-1}$, which is a strongly blueshifted graphene $2 \mathrm{D}$ peak. Due to the charge-neutrality as confirmed by ARPES and STS, this 
blueshift can be attributed to compressive strain ${ }^{10}$ in the graphene layer, which is presumably present due to a combination of the growth method and strain due to the opposite thermal expansion coefficients during the cooldown between the graphene and the substrate. Integrating the intensity of all the spectra over the range between 2700 and $2800 \mathrm{~cm}^{-1}$ shows where the 2D-peak originates. The intensity of the integrated signal is shown in Fig. S4(b) and shows a line pattern which corresponds to the geometry of the sample. The sidewall GNRs show a high intensity, whereas the buffer layer covering the rest of the sample does not contribute to the signal. Although the signal is convoluted with the size of the laser spot, which is roughly $800 \mathrm{~nm}$, the map shows a homogeneous Raman signal in large portions of the GNRs. 


\section{Tight binding calculations}

All tight binding calculations were performed within the Pybinding framework. 11 A onedimensional geometry was used, which is shown in Fig. S5. The unit cell spans the entire width of the ribbon, and translation symmetry in the longitudinal direction is applied. With the hopping integrals determined from the geometry, the Hamiltonian is set up, which is solved with the lapack algorithm from the Pybinding framework. The general Hamiltonian used can be written as

$$
\hat{H}=\sum_{<i, j>} t_{i j} c_{i}^{\dagger} c_{j}+\sum_{\ll i, j \gg} t^{\prime} e^{ \pm i \phi} c_{i}^{\dagger} c_{j}+H . C .
$$

where $\langle i, j\rangle(\ll i, j \gg)$ corresponds to (next) nearest neighbor hopping. $t_{i j}$ is the hopping integral between sites $i$ and $j$, which can vary when the ribbon is subjected to strain. $t^{\prime} e^{ \pm i \phi}$ is the Haldane next-nearest neighbor hopping term, with the sign in the exponent being positive (negative) for hopping between atoms of the A (B) sublattice and $\phi$ is the phase of the complex hopping, taken to be $\frac{\pi}{2}$ in all the calculations. The splitting for NNN hopping integrals is plotted in Fig. S6. Strain is introduced by defining a deformation profile $\epsilon_{y y}(y)$, which is then applied onto the geometry. Then, the hopping energy is calculated using a commonly used empirical expression:

$$
t_{i j}=t \exp \left[-\beta\left(\frac{d_{i j}}{a}-1\right)\right]
$$

where $\mathrm{t}$ represents the unstrained hopping integral with a value of $2.7 \mathrm{eV}$, a is the relaxed carbon-carbon distance of $1.42 \AA$ and $\beta=3.37 .12$

In order to compare splitting of the Haldane surface band with the experimental data, the expectation value of the transversal position $<\hat{y}_{n}>=<\psi_{n}|\hat{y}| \psi_{n}>$ of the surface state with band number $n$ was calculated across the BZ and plotted against the corresponding 
eigenenergies. Here, the transversal position operator is defined as:

$$
\hat{y}=\sum_{i} y_{i}|i><i|,
$$

with $\mathrm{i}$ indexing all sites.

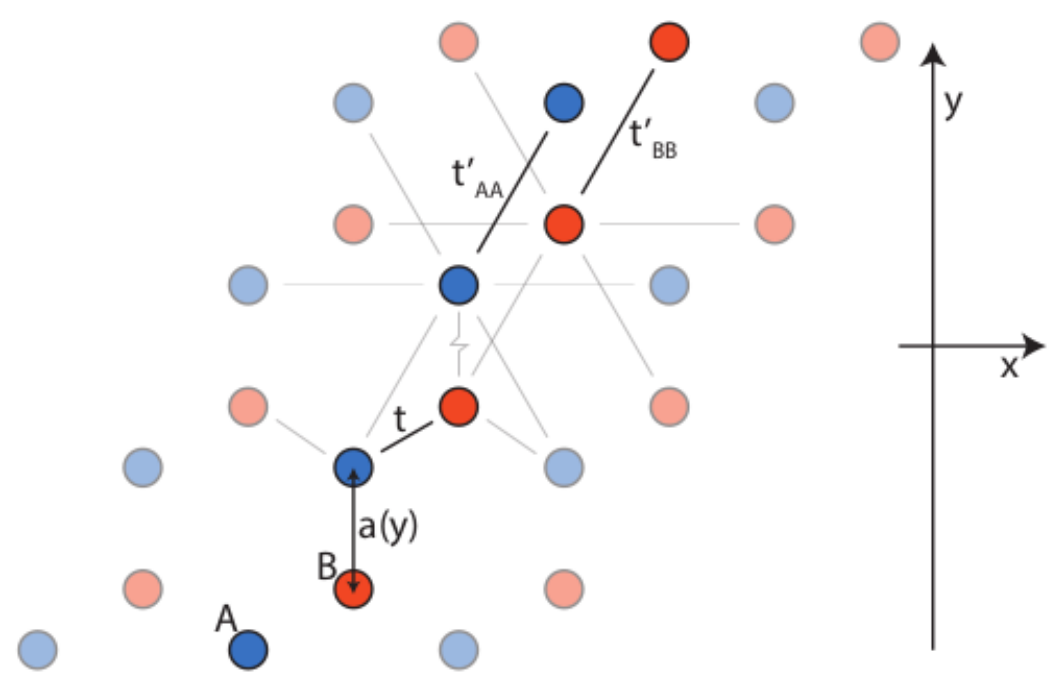

Figure S5: (color online) A schematic outline of the zz-GNR geometry used to perform tight binding calculations on. The unit cell spans the entire width of the ribbon and is periodic in the longitudinal $(\mathrm{x})$ direction, as is indicated with faded copies of the unit cell. The origin of the coordinate system is in the middle of the unit cell. A and B sublattices are indicated and colored blue and orange, respectively. Hoppings between neighboring sites are indicated with $t_{i j}$ and calculated taking into account the geometry. Furthermore, the complex next-nearest neighbor hoppings between the A and B sublattice sites are indicated.

Strain profile: Strain profiles are defined as a polynomial function of the transversal distance $y$ with a nonzero component only in the $\mathrm{y}$-direction. Different strain profiles were investigated. However, since a combination of homogeneous compressive strain and a strong strain gradient near the bent lower edge is expected, the strain profile was designed to resemble this:

$$
\epsilon_{y y}(y)=c+\alpha y^{9},
$$

where $\mathrm{c}$ denotes a constant strain and $\alpha$ determines the strength of the strain gradient near 
the edge. The value of the compressive strain used in the calculations - $3 \%$ - has been roughly estimated based on the blue-shift of the 2D peak of in the Raman spectra.

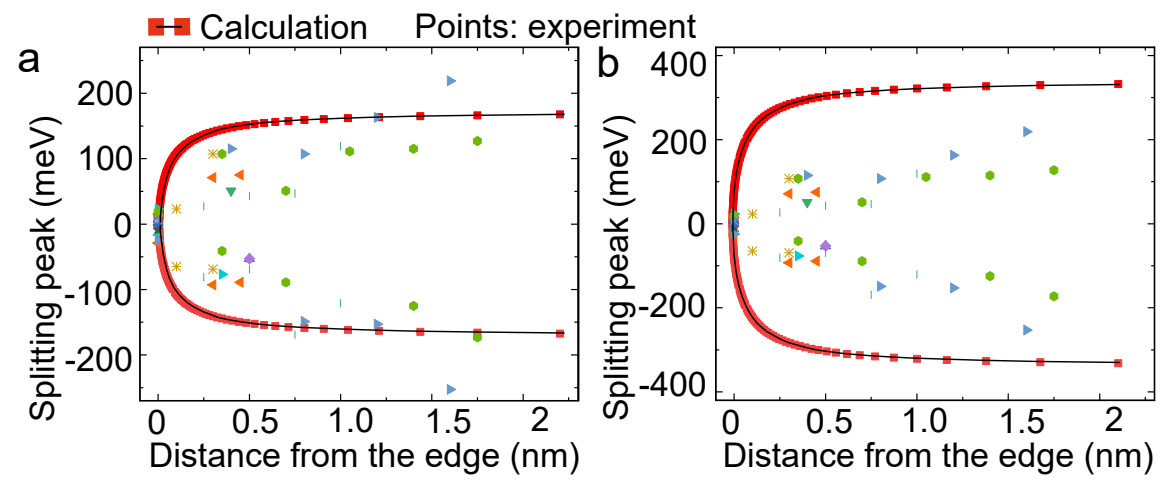

Figure S6: (color online) The splitting of the surface state for different NNN hopping strengths. a) Distance from the edge vs. energy of the peak STS data overlaid with the calculations $\left(t^{\prime}=100 \mathrm{meV}\right)$. b) Distance from the edge vs. energy of the peak STS data overlaid with the calculations $\left(t^{\prime}=200 \mathrm{meV}\right)$.

LDOS spectra: The LDOS spectra as a function of energy were calculated at different sites near the edge, using the expression

$$
D_{r}(E)=\frac{1}{\sigma \sqrt{2 \pi}} \sum_{n}\left|\psi_{n}(r)\right|^{2} e^{-\frac{\left(E_{n}-E\right)^{2}}{2 \sigma^{2}}}
$$

with $r$ indicating a site, $n$ indexing the bands, $E_{n}$ the band eigenvalues and $\sigma$ the broadening, set to $20 \mathrm{meV}$ in all LDOS calculations. These spectra, shown in Fig. S7, exhibit peaks corresponding to the Haldane surface state, splitting with increasing distance from the edge. Due to the surface state being fully sublattice polarized, only the LDOS spectra of the A (edge) sites are shown.

Chern number calculations: In order to prove the topology of the Haldane surface state found in the tight binding calculations, the first Chern number of the surface band was calculated. In order to perform the calculation, a plane wave description of the zz-GNR was used. The Berry curvature was calculated across the BZ according the its definition in reciprocal space:

$$
\Omega_{n}(\vec{k})=\nabla_{\vec{k}} i<u_{n}(\vec{k})\left|\nabla_{\vec{k}}\right| u_{n}(\vec{k})>,
$$




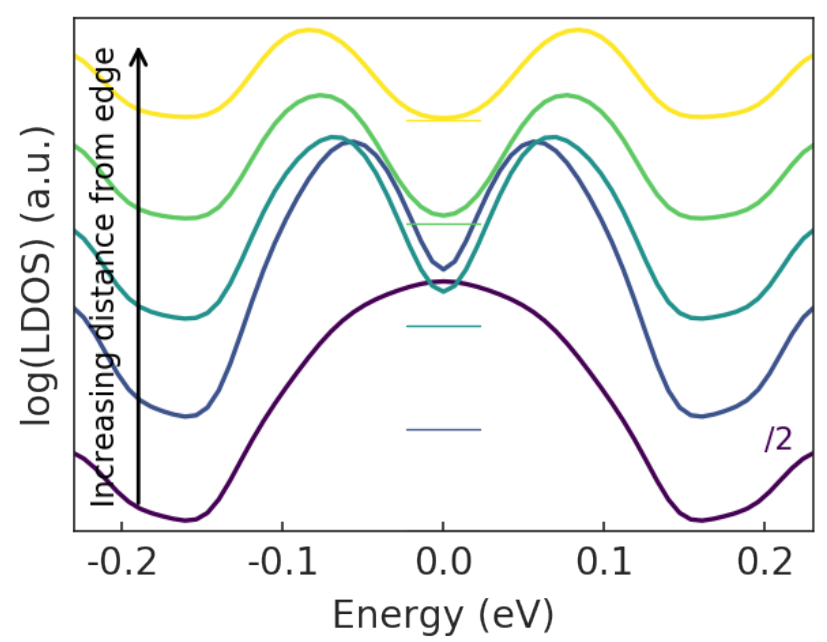

Figure S7: (color online) The local DOS at the five A-sublattice sites closest to the edge. Peaks in the spectra correspond to the Haldane surface state, splitting away from the Dirac point with increasing distance to the edge. The spectra correspond to next-nearest neighboring atoms, making the distance between the sites corresponding to each graph about $2.3 \AA$.

where $u_{n}(\vec{k})$ corresponds to the Bloch weight of the $\mathrm{n}^{\text {th }}$ state. Then, the Chern number of the $\mathrm{n}^{\text {th }}$ band is defined as:

$$
\mathcal{C}_{n}=-\frac{1}{2 \pi} \int_{B Z} \Omega_{n}(\vec{k}) d \vec{k},
$$

which is found to be -1 for the band corresponding to the surface state under the conditions used for the calculations presented in the main text. Best agreement with the experiment was obtained with the following parameters: hopping strengt, $t=2.7 \mathrm{eV}, \mathrm{NNN}$ hopping strength $t^{\prime}=0.1 \mathrm{eV}$, Haldane phase $\phi=\pi / 2$, compressive strain $c=0.03,9^{\text {th }}$ order strain $\alpha=-1.49 \times 10^{-11}$ and ribbon width $\mathrm{w}=20 \mathrm{~nm}$. The value for t' was acquired by fitting the splitting data to the values obtained experimentally, but due to the $\pi / 2$ Haldane phase, the consequent nextnearest neighbor hopping term in the model is a fully imaginary complex number. Coincidently, values in the same order of magnitude have been found more often from fits to first principle calculations for a second-nearest neighbor hopping term. ${ }^{13}$ As seen in separate convergence studies for the edge, $20 \mathrm{~nm}$ was wide enough in order to avoid any bulk-related confinement effects near the edge. The results obtained for the edge do not change if the width is doubled. Furthermore, for the calculation of the electronic gap in the 
next section, a $40 \mathrm{~nm}$ wide ribbon was used. 


\section{Bulk band gap and quantum well states}

Although mainly the electronic structure of the edge is considered due to its interesting feature, STS spectra were also recorded in the middle of the ribbon. This is called bulk because edge effects are not expected, but that does not mean that the electronic structure of bulk graphene is present there. While the band structure of a zigzag GNR could be discussed to be metallic, the bands that do cross the Fermi level correspond to localized surface states. Due to slight electron confinement in the transversal direction, a bulk band gap is opened at the K-points. This gap has been observed to be $130 \mathrm{meV}$ from STS spectra taken in the middle of the ribbon, as is shown in Fig. S8. This is in agreement with a gap of $120 \pm 10 \mathrm{meV}$ that was found in the tight binding model, as is shown in Fig. S9. The spectra shown in Fig. S8 show much clearer the electronic gap and in addition also expected intensity variations due to quantum well states compared to the spectra shown in the main text (cf. Fig. 2d). The spectra shown in Fig. S8, which are in better agreement with the TB calculations, were seen only occasionally on a few ribbons. Apparently, in most of the STS measurements these quantum features are averaged out, suggesting that the confinement conditions are spatially varying and might be altered in addition also by local variation of strain within the GNR (cf.Fig. 2c).

Although the value of $120 \mathrm{meV}$ may seem rather large for an electron confinement gap, such values have been reported numerically before. ${ }^{16}$ It can be understood by considering that due to the finite size, the band structure of the ribbon is just a projection of the graphene band structure onto a discretized Brillouin zone. Hence, the distance between the second conductance- and valence bands just corresponds to the band gap at a small distance away from the K-point in the band structure of an infinite sheet. This distance is given by the distance between two adjacent $\mathrm{k}$-values in the discretized Brillouin zone: $k_{d}=\frac{4 \pi}{\sqrt{3} a N}$. Assuming a linear dispersion near the $\bar{K}$-points and $\nu_{f}=10^{6} \mathrm{~m} / \mathrm{s}$, the expression for the 
bulk gap would then be given by:

$$
\Delta_{b u l k}=\hbar \nu_{f} k_{d} \approx \frac{4.77}{W}[\mathrm{eV} \cdot \mathrm{nm}]
$$

which corresponds to a bulk gap of $\Delta_{b u l k} \approx 120 \mathrm{meV}$ for a ribbon with a width of $a N \approx 40 \mathrm{~nm}$.

The gap may be interpreted also the result of a so-called phonon-assisted inelastic tunneling gap, which is a hallmark for suspended graphene. Here, the momentum of the tunneling electron to/from the $\bar{K}$-point is mediated by a phonon with an excitation energy of $63 \mathrm{meV} .17$ However, there are arguments against this interpretation. For instance, for an atomically sharp tip the electron momentum is restricted by the uncertainty principle.

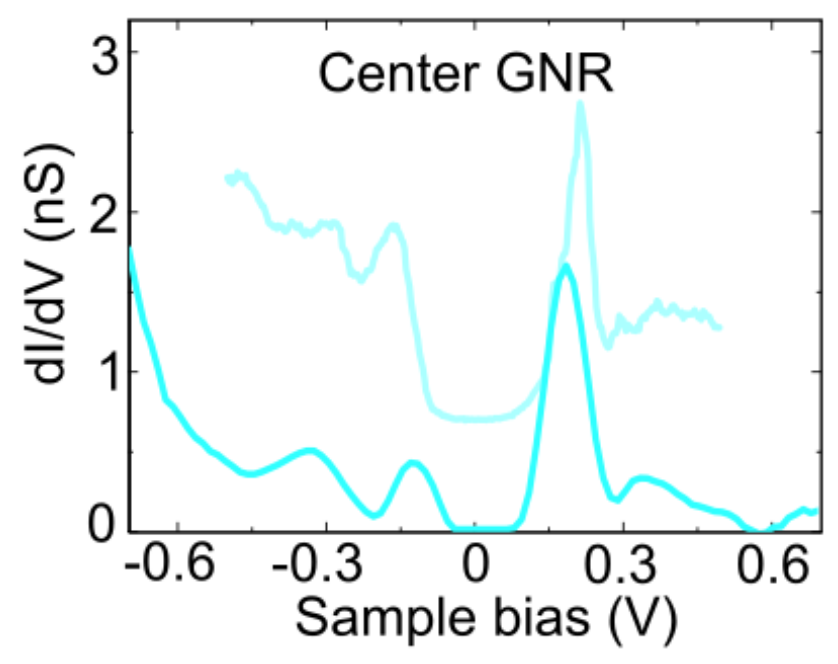

Figure S8: STS spectra taken in the center of the zz-GNR revealing clearly a gap around zero bias as well as peaks which are associated with quantum well state. A gap size of around $130 \mathrm{meV}$ can be deduced in good agreement with eq. 8 .

Fig. S10 reveals the band structure with and without disorder including an energy broadening of $10 \mathrm{mV}$. The bands are weighted with their real-space cross-section, thus the surface states are not or hardly visible as they are only extending $1 \mathrm{~nm}$ into the ribbon. While the band gap is almost not affected, the high intensity $\pi$ - bands as well as the states in the area in between are filled due to disorder. Therefore it is likely that the QWS states are not visible in the ARPES K-cross-section as they do have a different transversal momentum. 


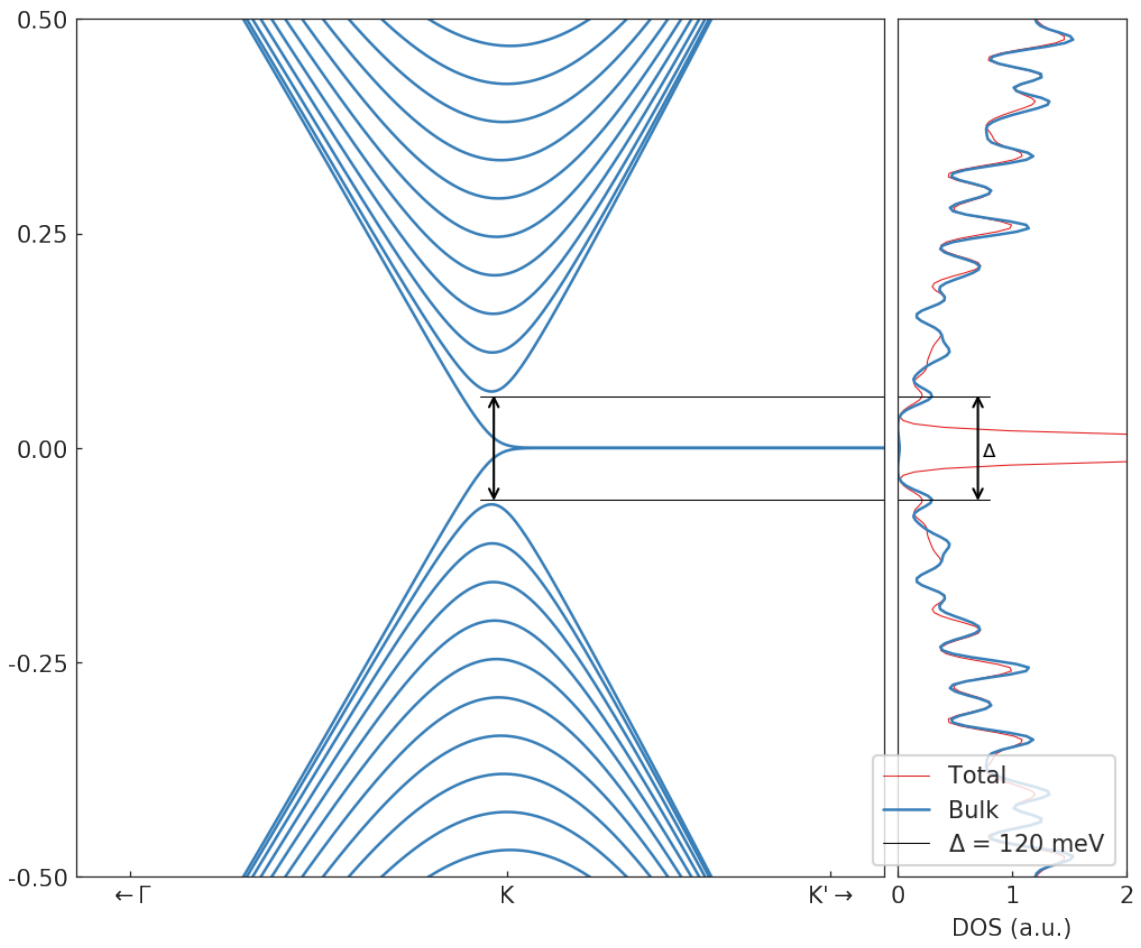

Figure S9: The left graph shows one Dirac cone from the band structure of a $40 \mathrm{~nm}$ wide graphene nanoribbon. In the right graph, the corresponding total DOS of the structure is shown, as well as the DOS calculated in the exact middle of the ribbon. Whereas the total DOS exhibits a large peak at $E_{F}$, the LDOS in the bulk of the ribbon exhibits a band gap of $\Delta_{b u l k}=120 \mathrm{meV}$, which is introduced due to finite electron confinement in the transversal direction of the ribbon. 


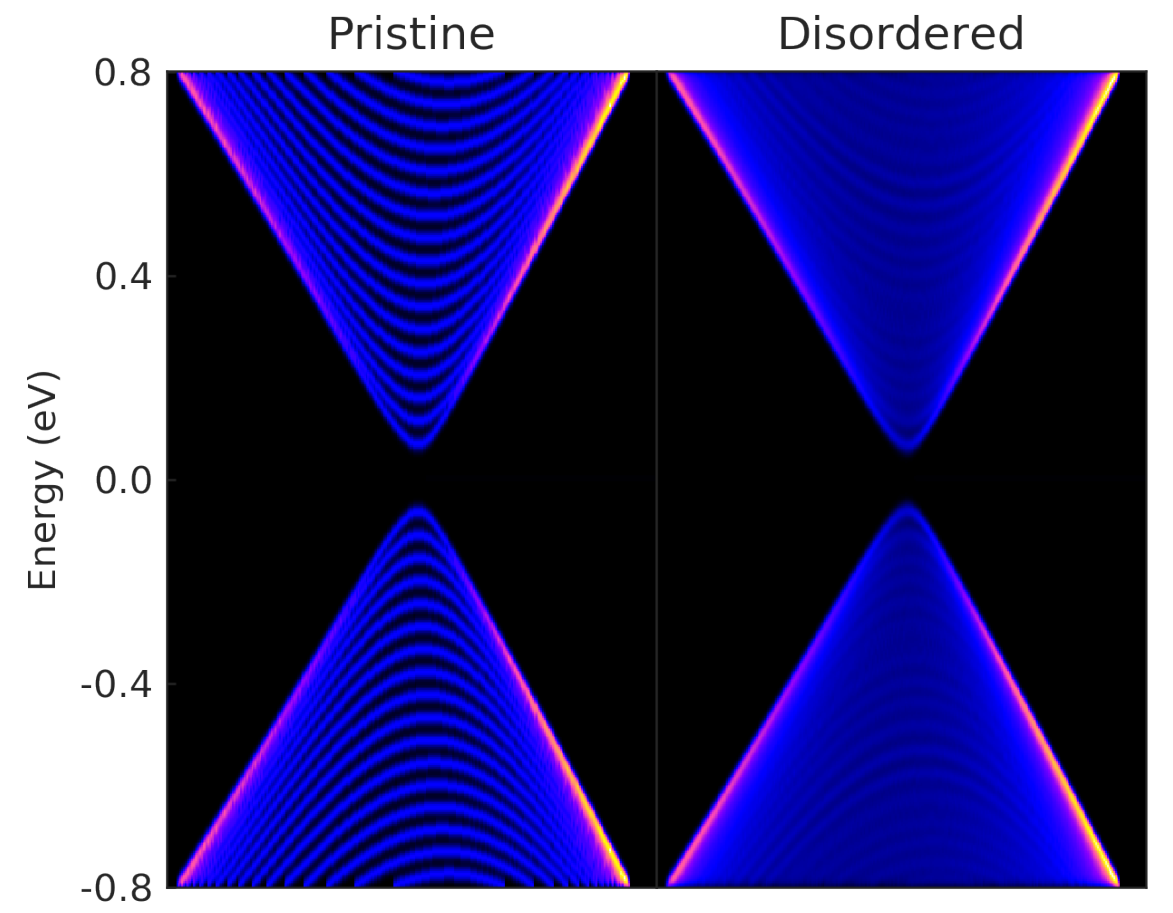

Figure S10: Band structure of a $40 \mathrm{~nm}$ wide pristined (left) and disordered (right) zz-GNR. While the band gap is almost not affected by disorder, the QWS features are strongly smeared out. Note, the bands are weighted with the real-space cross-section of the corresponding states, hence the surface state is not visible. 


\section{Transmission electron microscopy (TEM) measurements}

As outlined, the bonding of the lower zz-GNR edge is important. In order to characterize the lower edge, first transmission electron microscopy (TEM) experiments were performed. Lamella structures of the graphene/ $\mathrm{SiC}(0001)$ samples for cross-sectional scanning transmission electron microscopy (STEM) were prepared utilizing different focused ion beam (FIB) systems (Helios G4 UC and Scios 2 dual beam, Thermo Fisher Scientific) both using Ga ion sources. Prior to the FIB preparation, the samples were coated with an approximately 200 $\mathrm{nm}$ thick layer of amorphous carbon followed by the deposition of a $2 \mu \mathrm{m}$ thick layer of platinum. The lamellas were cut out with a thickness of a few microns. Electron transparency was finally reached by thinning the lamellas down to about $30 \mathrm{~nm}$ around the interface area by subsequently using ions of $30 \mathrm{keV}, 5 \mathrm{keV}$, and $2 \mathrm{keV}$ for polishing under an angle of approximately $1^{\circ}$. The samples were characterized in detail by means of a double $\mathrm{C}_{s^{-}}$ corrected STEM (ThemisZ, Thermo Fisher Scientific) operating for our studies at a primary electron energy of $80 \mathrm{keV}$ in order to prevent knock-on damage in the graphene films and $\mathrm{SiC}$ substrate. $\frac{18119}{10}$

The electronic properties of the ribbons at its lower edge were investigated in more detail by electron energy loss spectroscopy (EELS). In order to improve the signal-to-noise ratio, the spectra were averaged along the $[11 \overline{2} 0]$ direction over a spatial window of approximately $1.5 \mathrm{~nm}$ at different positions across the lamella structure. The spectra reveal two main loss features at the C-K edge: $\stackrel{20}{2}$ The loss at approximately $287 \mathrm{eV}$ refers to the $1 \mathrm{~s} \rightarrow \pi^{*}$-transition, while the one at around $293 \mathrm{eV}$ is assigned to the $1 \mathrm{~s} \rightarrow \sigma^{*}$-transition as well as transitions into energetically higher $\pi$ and $\sigma$ orbitals. ${ }^{21}$ Thus, these losses come along with the presence of $\pi$ and $\sigma$ bonds in carbon and are indicative for $\mathrm{sp}^{2}$ hybridization, as in graphene, or $\mathrm{sp}^{3}$ hybridization like for $\mathrm{C}$ in $\mathrm{SiC}$. A corresponding color-coded EELS-map of the lower part of the GNR is shown in Fig. S11 a, showing clearly a suppression of the $\mathrm{sp}^{2}$-character of the ribbon at the root point.

In Fig. S11b) we show further STEM data from zz-GNRs grown on SiC facets. In contrast 
to the former one, this structure was heated too long, thus multilayer GNRs were grown. However, also these merge into the $\mathrm{SiC}$ substrate inducing a considerable strain in the vicinity of the lower edge of the GNR. This growth mode seems to be characteristic and was reported also on $\mathrm{SiC}(0001)$ surfaces with atomic sized steps. 22
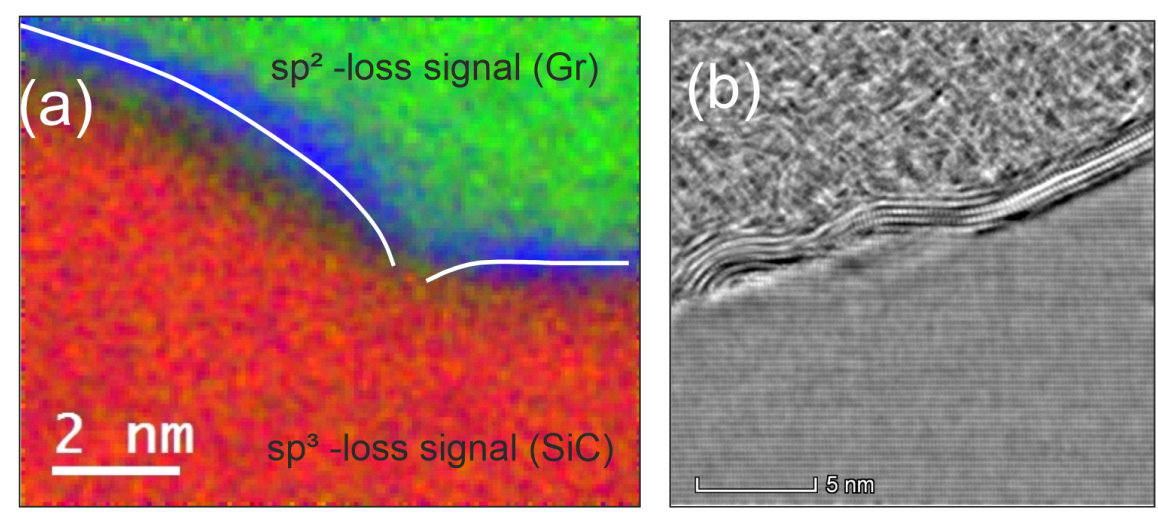

Figure S11: (color online) (a) EELS map of the zz-GNR shown in the main text. The red and blue colors refer to the $\mathrm{sp}^{2}$ - and $\mathrm{sp}^{3}$-specific loss channels, respectively. The discontinuity within the $\mathrm{sp}^{2}$ state seen between the GNR on the facet and the buffer layer on the flat part is referred to the merging of the ribbon into the $\mathrm{SiC}$. The white lines are guides to the eye. (b) STEM image of a different GNR/SiC system, which were overheated, thus multiple ribbons were formed. However, all of them merge into the $\mathrm{SiC}$ substrate. 


\section{References}

(1) Riedl, C.; Coletti, C.; Starke, U. Structural and electronic properties of epitaxial graphene on $\mathrm{SiC}(0001)$ : a review of growth, characterization, transfer doping and hydrogen intercalation. Journal of Physics D: Applied Physics 2010, 43, 374009.

(2) Horn-von Hoegen, M. Growth of semiconductor layers studied by spot profile analysing low energy electron diffraction. Zeitschrift für Kristallographie 1999, 214, 591.

(3) Meyer zu Heringdorf, F.-J.; Horn-von Hoegen, M. Reciprocal space mapping by spot profile analyzing low energy electron diffraction. Review of Scientific Instruments $\mathbf{2 0 0 5}$, $76,085102$.

(4) Klein, C.; Nabbefeld, T.; Hattab, H.; Meyer, D.; Jnawali, G.; Kammler, M.; zu Heringdorf, F.-J. M.; Golla-Franz, A.; Müller, B. H.; Schmidt, T.; Henzler, M.; Horn-von Hoegen, M. Lost in reciprocal space? Determination of the scattering condition in spot profile analysis low-energy electron diffraction. Review of Scientific Instruments 2011, 82, 035111.

(5) Stöhr, A.; Baringhaus, J.; Aprojanz, J.; Link, S.; Tegenkamp, C.; Niu, Y.; Zakharov, A. A.; Chen, C.; Avila, J.; Asensio, M. C.; Starke, U. Graphene Ribbon Growth on Structured Silicon Carbide. Annalen der Physik 2017, 529, 1700052.

(6) Ristein, J.; Mammadov, S.; Seyller, T. Origin of Doping in Quasi-Free-Standing Graphene on Silicon Carbide. Phys. Rev. Lett. 2012, 108, 246104.

(7) Riedl, C.; Coletti, C.; Iwasaki, T.; Zakharov, A. A.; Starke, U. Quasi-Free-Standing Epitaxial Graphene on SiC Obtained by Hydrogen Intercalation. Phys. Rev. Lett. 2009, $103,246804$.

(8) Karakachian, K.; Nhung Nguyen, T.; Aprojanz, J.; A.A., Z.; Yakimova, R.; Rosenzweig, P.; Polley, C.; Balasubramanian, T.; ; Tegenkamp, C.; Power, S.; Starke, U. One- 
dimensional confinement and width-dependent bandgap formation in epitaxial graphene nanoribbons. Nature Comm. 2020, 11, 6380.

(9) Miettinen, A. L.; Nevius, M. S.; Ko, W.; Kolmer, M.; Li, A.-P.; Nair, M. N.; Kierren, B.; Moreau, L.; Conrad, E. H.; Tejeda, A. Edge states and ballistic transport in zigzag graphene ribbons: The role of SiC polytypes. Phys. Rev. B 2019, 100, 045425.

(10) Mueller, N. S. et al. Evaluating arbitrary strain configurations and doping in graphene with Raman spectroscopy. 2D Materials 2018, 5 .

(11) Moldovan, D.; And̄elković, M.; Peeters, F. pybinding v0.9.4: a Python package for tight- binding calculations. 2017; https://doi.org/10.5281/zenodo.826942.

(12) Pereira, V. M.; Castro Neto, A. H.; Peres, N. M. Tight-binding approach to uniaxial strain in graphene. Physical Review B - Condensed Matter and Materials Physics 2009, $80,1-8$.

(13) Kundu, R. Tight-Binding Parameters for Graphene. Mod. Phys. Lett. B 2011, 25, 163-173.

(14) Reich, S.; Maultzsch, J.; Thomsen, C.; Ordejón, P. Tight-binding description of graphene. Phys. Rev. B 2002, 66, 035412.

(15) Tran, V.-T.; Saint-Martin, J. o.; Dollfus, P.; Volz, S. Third nearest neighbor parameterized tight binding model for graphene nano-ribbons. AIP Advances 2017, 7, 075212.

(16) Areshkin, D. A.; Gunlycke, D.; White, C. T. Ballistic transport in graphene nanostrips in the presence of disorder: Importance of edge effects. Nano Letters 2007, 7, 204-210.

(17) Levy, N.; Burke, S.; Meaker, K. L.; Panlasigui, M.; Zettl, A.; Guinea, F.; Castro Neto, A. H.; Crommie, M. Strain-Induced PseudoâĂŞMagnetic Fields Greater Than 300 Tesla in Graphene Nanobubbles. Science 2010, 329, 544-547. 
(18) Egerton, R. F.; McLeod, R.; Wang, F.; Malac, M. Basic questions related to electroninduced sputtering in the TEM. Ultramicroscopy 2010, 110, 991-997.

(19) Egerton, R. F. Choice of operating voltage for a transmission electron microscope. Ultramicroscopy 2014, 145, 85-93.

(20) Gruschwitz, M.; Schletter, H.; Schulze, S.; Alexandrou, I.; Tegenkamp, C. Epitaxial graphene on 6H-SiC(0001): Defects in SiC investigated by STEM. Phys. Rev. Materials 2019, 3, 094004.

(21) Nicotra, G.; Ramasse, Q. M.; Deretzis, I.; La Magna, A.; Spinella, C.; Giannazzo, F. Delaminated Graphene at Silicon Carbide Facets: Atomic Scale Imaging and Spectroscopy. ACS Nano 2013, 7, 3045-3052.

(22) Norimatsu, W.; Kusunoki, M. Formation process of graphene on SiC (0001). Physica E: Low-dimensional Systems and Nanostructures 2010, 42, 691 - 694, 18th International Conference on Electron Properties of Two-Dimensional Systems. 